\title{
Compromised vertebral structural and mechanical properties associated with progressive kidney disease and the effects of traditional pharmacological interventions
}

\author{
Christopher L. Newman ${ }^{1}$, Neal X. Chen ${ }^{2}$, Eric Smith ${ }^{1}$, Mark Smith ${ }^{1}$, Drew Brown ${ }^{1}$, Sharon \\ M. Moe ${ }^{2,3}$, and Matthew R. Allen ${ }^{1,2}$ \\ ${ }^{1}$ Department of Anatomy and Cell Biology, Indiana University School of Medicine, Indianapolis, \\ IN, United States. \\ ${ }^{2}$ Department of Medicine, Indiana University School of Medicine, Indianapolis, IN, United States. \\ ${ }^{3}$ Roudebush VA Medical Center, Indianapolis, IN, United States.
}

\section{Abstract}

Background/Aims-Patients with chronic kidney disease mineral and bone disorder (CKDMBD) have a significantly higher vertebral and non-vertebral fracture risk than the general population. Several preclinical models have documented altered skeletal properties in long bones, but few data exist for vertebral bone. The goal of this study was to examine the effects of progressive CKD on vertebral bone structure and mechanics and to determine the effects of treatment with either bisphosphonates or anti-sclerostin antibody in groups of animals with high or low PTH.

\begin{abstract}
Methods-Animals with progressive kidney disease were left untreated, treated with calcium to lower PTH, zoledronic acid to lower remodeling without affecting PTH, anti-sclerostin antibody, or anti-sclerostin antibody plus calcium. Non-diseased, untreated littermates served as controls. Vertebral bone morphology (trabecular and cortical) and mechanical properties (structural and material-level) were assessed at 35 weeks of age by microCT and mechanical testing, respectively.

Results-CKD with high PTH resulted in 6-fold higher bone formation rate, significant reductions in the amount of trabecular and cortical bone, and compromised whole bone mechanical properties in the vertebra compared to normal animals. Treatments that reduced bone remodeling were effective in normalizing vertebral structure and mechanical properties only if the treatment reduced serum PTH. Similarly, treatment with anti-sclerostin antibody was effective in enhancing bone mass and mechanical properties but only if combined with PTH-suppressive treatment.
\end{abstract}

\footnotetext{
(C) 2015 Published by Elsevier Inc.

Send Correspondence to: Matthew R. Allen, PhD Dept. of Anatomy and Cell Biology, MS 5035 Indiana University School of Medicine 635 Barnhill Dr. Indianapolis, IN 46202 Tel: 317-274-1283 FAX: 317-278-2040 matallen@iupui.edu.

Publisher's Disclaimer: This is a PDF file of an unedited manuscript that has been accepted for publication. As a service to our customers we are providing this early version of the manuscript. The manuscript will undergo copyediting, typesetting, and review of the resulting proof before it is published in its final citable form. Please note that during the production process errors may be discovered which could affect the content, and all legal disclaimers that apply to the journal pertain.
} 
Conclusions-CKD significantly altered both cortical and trabecular bone properties in the vertebra resulting in compromised mechanical properties and these changes can be normalized by interventions that involve reductions in PTH levels.

\section{Keywords}

spine; zoledronic acid; CKD-MBD; anti-sclerostin antibody; PTH; chronic kidney disease

\section{INTRODUCTION}

Patients with chronic kidney disease-mineral and bone disorder (CKD-MBD) have a significantly higher fracture risk than the general population [1-3]. This population also displays differences in fracture rates between long bones and vertebrae [3], suggesting that these two skeletal sites may be differentially affected by the disease. A study of Japanese men on dialysis who underwent screening lumbar spine imaging studies demonstrated that $20.9 \%$ of prevalent dialysis patients had evidence of spine fractures [4]. High resolution CT data have revealed significant increases in cortical porosity in the distal limbs with variable responses in trabecular bone [5]. Because vertebral elements are primarily composed of trabecular bone, the influence of secondary hyperparathyroidism on these sites could be potentially different than in long bone cortices [6]. Furthermore, the thin cortical shell of the vertebrae bears nearly $50 \%$ of the load [7]; thus, cortical bone changes at this site would also have dramatic effects on mechanical properties and fracture risk

Several animal models of CKD have revealed significant detriments in the biomechanical properties of long bones [8-10]. For example, rodent models indicate that animals with CKD exhibit lower strength and stiffness compared to their normal counterparts [11-13]. While much of the decline in mechanical integrity can be attributed to structural changes resulting from high parathyroid hormone (PTH) and high turnover rates, recent studies indicate that bone quality also plays a role [9]. Unfortunately, vertebral bone in CKD models has yet to be examined at any of these levels. The goal of this study was to assess the effects of progressive CKD on vertebral bone structure and mechanics and to determine the effects of treatment with either anti-remodeling medications (bisphosphonates) or anabolic treatments (anti-sclerostin antibody). We hypothesized that a significant phenotype would exist in the vertebrae of CKD animals and that treatment would restore structural mechanical properties but not the material-level mechanical properties.

\section{METHODS}

\section{Animal model}

$\mathrm{Cy} /+$ rats exhibit the progressive onset of polycystic kidney disease due to transmission of an autosomal dominant missense mutation in the geneAnks6, which codes for the protein SamCystin [14-16]. Anks6 has been shown to be important in childhood recessive cystic kidney disease although heterozygote parents have no manifestations [17-19]. The course of kidney disease progression in the $\mathrm{Cy} /+$ rats parallels the course of human CKD-Mineral Bone Disorder (CKD-MBD) [16]. A colony of these animals is maintained at the Indiana 
University School of Medicine. All procedures were reviewed and approved by the Indiana University School of Medicine Institutional Animal Care and Use Committee.

\section{Experimental design}

The animals described in this work were part of a large experiment that included numerous treatment and control groups. Male $\mathrm{Cy} /+$ rats began the study at 25 weeks of age at which time animals were fed a casein-based diet (Purina AIN-76A, Purina Animal Nutrition, Shreevport, LA, USA); $0.53 \% \mathrm{Ca}$ and $0.56 \% \mathrm{P}$ ) in order to accentuate the disease. Subsets of $\mathrm{Cy} /+$ animals were divided into the following groups (Figure 1):

Controls (CKD)-These animals were left untreated. Based on the previously described phenotype these animals have high PTH and high bone turnover [11,20].

Calcium (CKD-Ca)—These animals were treated with calcium-supplemented water (3\%) starting at 30 weeks of age. Based on the previously described phenotype these animals have low PTH and low bone turnover $[11,20]$.

Zoledronic acid (CKD-Z)—These animals were treated with zoledronic acid (20 $\mathrm{ug} / \mathrm{kg} / \mathrm{BW}$ as a single subcutaneous injection at 30 weeks of age). Based on the previously described phenotype these animals have high PTH and low bone turnover [11,20].

Zoledronic acid was chosen as a representative bisphosphonate to study in CKD as it has an infrequent dosing schedule (1x per year in post-menopausal osteoporosis treatment) and has high affinity for the bone matrix thus likely to impart persistent treatment effects.

Anti-sclerostin antibody (CKD-SCL) - These animals were treated with anti-sclerostin antibody (Scl-Ab; $100 \mathrm{mg} / \mathrm{kg} /$ week starting at 30 weeks of age). Scl-AB was provided by Novartis Institutes for BioMedical Research, Novartis Pharma AG. Based on the previously described phenotype these animals have high PTH and high bone turnover [11,20]. Antisclerostin antibody was chosen as it has emerged as a promising potential anabolic drug treatment.

Anti-sclerostin antibody plus calcium (CKD-SCL-Ca): These animals were treated with anti-sclerostin antibody and calcium (3\% in water). Based on the previously described phenotype these animals have low PTH and high bone turnover [11,20].

Normal (NL)_A group of non-diseased male littermates were untreated.

All animals were sacrificed at 35 weeks of age. For all experiments, serum biochemistries and long bone phenotypes have been previously reported in various publications, yet are repeated in the results when deemed necessary $[11,13,20]$.

\section{Dynamic Histomorphometry}

In order to describe the basal remodeling phenotype of the vertebra in NL and CKD animals, the third lumbar vertebra of animals from a separate experiment [13] was processed for undecalcified histomorphometry. The vertebral arch was removed, and the body was embedded in PMMA for sectioning. Thin sections ( $4 \mathrm{um})$ were cut and left unstained for 
analysis. Briefly, a $2 \mathrm{~mm}^{2}$ region of interest in the caudal region of the vertebra cranial to the growth plate was used for the analysis. Primary measurements were obtained and used for the derived parameters mineralizing surface/bone surface (MS/BS), mineral apposition rate (MAR), and bone formation rate/bone surface (BFR) as previously described [11] using standard nomenclature [21].

\section{Computed tomography}

Morphological parameters of the fourth lumbar vertebrae from all experiments were assessed using high-resolution micro-CT (Skyscan 1172). Bones were wrapped in parafilm to prevent drying during the scanning. Scans were obtained using an x-ray source, set at $60 \mathrm{kV}$ with a $12-\mu \mathrm{m}$ pixel size.

Images were reconstructed and analyzed using standard Skyscan software (NRecon and CTAn, respectively). Trabecular regions of interest were manually drawn to isolate it from the cortical bone between the cranial and caudal growth plates. Bone volume/tissue volume (BV/TV), trabecular number (Tb.N), and trabecular thickness (Tb.Th) were computed according to previously published recommendations [22]. A single slice at $75 \%$ of the total vertebral length (from cranial to caudal) was used for the assessment of cortical thickness as this represents a region devoid of zygapophyseal attachment. Bone area was assessed at $25 \%, 50 \%$, and $75 \%$ of the total height for normalization of mechanical properties.

\section{Mechanics}

Structural mechanical properties and apparent material properties were assessed by uniaxial compression testing of the L4 vertebra from all 3 experiments. At harvest, the vertebrae were wrapped in saline-soaked gauze and frozen at -20 degrees. Bone height was assessed from microCT images. Prior to mechanical testing, the vertebral arch and the endplates were removed using an Isomet saw in order to create parallel surfaces (beneath the growth plates) for compression testing. Samples were loaded in displacement control at $0.5 \mathrm{~mm} / \mathrm{min}$. Load and displacement data were collected at $10 \mathrm{~Hz}$ and converted to stress and strain data from the initial sample height and the average bone area of 3 slices $(25 \%, 50 \%$, and $75 \%$ slices of the initial height of the sample). Load-displacement data were used to compute ultimate load, stiffness, and energy to failure (with failure defined as the point ultimate load first drops off), while apparent material properties were derived from the stress-strain data and used to calculate ultimate stress, elastic modulus, and toughness to the point of the ultimate stress [23].

\section{Statistics}

Statistics were carried out on select groups in order to answer a priori questions related to 1) basal phenotype of the CKD model, 2) the effects of anti-remodeling treatment with and without altered PTH levels, and 3) the effects of anabolic treatment with and without altered PTH levels (Figure 1). Comparisons were made using student's t-tests or one-way ANOVA followed by Fisher protected least significant difference post hoc tests. P values were set at $<0.05$. Data are reported as means and standard deviations. 


\section{RESULTS}

CKD with high PTH leads to high remodeling rate, bone loss, and compromised structural and material mechanical properties

Select biochemistries and animal body weights are presented in Table 1. PTH levels in the CKD animals were over 10-fold higher than normal animals (Figure 1). Histological assessment of vertebral trabecular bone revealed that mineral apposition rate was more than doubled $(\mathrm{NL}=1.21$; $\mathrm{CKD}=2.98)$, mineralizing surface/bone surface was $2.5 \mathrm{x}$ higher $(\mathrm{NL}$ $=10.5 ; \mathrm{CKD}=24.5)$ and bone formation was 6 -fold higher in CKD animals compared to normal (Figure 2A). Thus, the untreated CKD model is one of high PTH and high remodeling as illustrated in the photomicrographs (Figure 2F-G).

Vertebral microCT analyses showed that cortical thickness (-17\%) and trabecular BV/TV $(-30 \%)$ were both significantly lower in CKD compared to normal (Figure $2 B \& \mathbf{C}$ and $\mathbf{H}-$ K; Table 2). Ultimate force (-34\%), stiffness $(-21 \%)$, and energy to failure $(-42 \%)$ were all significantly lower in CKD compared to NL (Figure 2D \&E; Table 2). Calculation of material properties revealed significant lower ultimate stress (-24\%) and toughness $(-34 \%)$, but not modulus, in CKD animals (Table 2).

\section{Low turnover with suppression of PTH normalizes vertebral bone morphology and mechanical properties}

CKD animals treated with calcium (CKD-Ca), which reduced PTH and tibial bone suppresses bone remodeling [20], had similar vertebral cortical thickness but significantly higher trabecular BV/TV (+15\%), compared to normal, non-diseased animals (Figure 3A\&B; Table 2). Cortical thickness and BV/TV in CKD-Ca were both significantly higher than CKD animals. Ultimate load and energy to failure of CKD-Ca vertrebrae were equivalent to NL (and higher than CKD) with the exception of stiffness, which was not significantly different among groups (Figure 3C\&D; Table 2). Ultimate stress, modulus, and toughness of CKD-Ca were all equal or higher than NL animals (Table 2) and all higher than CKD.

In animals treated with zoledronic acid (CKD-Z), which suppresses bone remodeling without lowering PTH [20], the results were in stark contrast to CKD-Ca. Cortical thickness $(-16 \%)$ and trabecular BV/TV $(-18 \%)$ were both significantly lower than NL and CKD-Ca (Figure 3A\&B; Table 2). Ultimate force (-28\%) was significantly lower in CKD-Z compared to NL, while stiffness and energy to failure were not different (Figure 3C\&D;

Table 2). Ultimate stress and modulus were lower than NL, while all three material properties were significantly lower than CKD-Ca (Table 2).

\section{High turnover with suppression of PTH, but not without, enhances vertebral morphology and vertebral mechanical properties}

CKD animals with high PTH treated with anti-sclerostin antibody, previously shown to not alter PTH or bone remodeling relative to untreated CKD [20], had significantly lower cortical thickness $(-11 \%)$, trabecular bone volume (-27\%), and ultimate load $(-32 \%)$, and stiffness (-54\%) compared to NL (Figure 4A-C; Table 2). Ultimate stress and modulus 
were both significantly lower than NL (Table 2). Overall, the vertebral phenotype of the CKD-Scl animals closely resembled the CKD animals with the exception of stiffness/ modulus (which were lower) and energy to failure/toughness (which were higher).

When anti-sclerostin antibody was combined with calcium (CKD-SCL-Ca), effectively reducing PTH and normalizing remodeling to NL levels [20], the results were dramatically different than anti-sclerostin alone. Cortical thickness was equivalent to NL animals while BV/TV (+15\%) was significantly higher than NL, equivalent to CKD-Ca (Figure 4A\&B;

Table 2). Ultimate load (+25\%) was significantly higher than all other treatments while energy to failure $(+51 \%)$ was significantly higher than all treatments except CKD-Ca (Figure 4C\&D; Table 2) while material properties were all comparable to NL animals, higher than most other groups (Table 2).

\section{DISCUSSION}

The goal of this study was to assess the vertebral phenotype of CKD given the high clinical risk of vertebral fracture in this patient population $[4,24]$ and the paucity of preclinical data on spine changes with CKD. In this progressive CKD animal model we found 1) elevations in endogenous PTH were associated with high turnover bone disease, compromised cortical and trabecular bone mass, and reduced mechanical properties (both structural and material) of the vertebra and 2) treatment of the vertebral phenotype with either anti-resorptive or anabolic therapies was only effective, from a mechanical standpoint, if serum PTH was reduced.

CKD patients have a higher risk of fracture compared to non-CKD patients [25]. Changes to long bones have been the central focus of most clinical work due to the advances in highresolution peripheral imaging which has yielded rich information regarding changes to cortical/trabecular bone. Most notably these studies have shown that changes to the cortical bone are dramatic [5] and more severe than are typically observed with post-menopausal osteoporosis (which affects trabecular bone to a greater degree, at least initially). The risk of vertebral fracture is also increased in CKD patients [4,24,26,27], yet little information exists regarding changes to bone mass/architecture at this site. The distribution of cortical and trabecular bone in the vertebra differs significantly from long bones and although cortical bone still contributes to the mechanical integrity of the spine [7], it is possible that the intricacies of bone loss may differ from long bones. The paucity of clinical studies focused on the spine is understandable due to imaging limitations. However for reasons that are unclear this extends to preclinical studies where few studies have examined vertebral bone [8].

The current study showed dramatic loss of trabecular bone mass and thinning of the cortical shell in the vertebra of CKD animals with high PTH, irrespective of whether they were untreated, treated with bisphosphonate, or treated with anti-sclerostin antibody. These results contrast with the long bone phenotype of these same animals where cortical porosity was higher than normal but trabecular BV/TV was not positively affected in high PTH animals treated with these agents [20] (Figure 5). In high-PTH CKD animals, both vertebra and proximal tibia trabecular bone had high bone formation rate, suggesting that simply having 
high remodeling does not explain the divergent trabecular BV/TV. In conditions where PTH is normalized, using calcium supplementation, both trabecular and cortical bone area normalized (or enhanced) compared to non-CKD animals. While additional work will be necessary to elucidate why long bones and vertebra differentially respond in the setting of CKD, it raises concern about the clinical use of iliac crest bone biopsies as an assessment of trabecular bone, especially in assessing bone volume [28-32]. It may be that iliac crest biopsy changes are more reflective of long bone trabecular responses than they are of the vertebra. This is supported by an previous study in renal transplant patients that showed no change in iliac crest biopsy cancellous BV/TV after 6 months while spine, but not radius, BMD changed significantly during this same time frame [33].

The structural mechanical phenotype of the vertebrae closely follows that of the long bones in these same animals, where compromised ultimate load, stiffness, and energy to fracture existed in animals with high PTH, even if treated with the potent anti-remodeling agent zoledronic acid or the potent anabolic anti-sclerostin antibody [20]. Treatment with calcium, to lower PTH, normalized vertebra ultimate load and energy to failure, both parameters that have functional significance for fracture resistance [34]. In animals where reduced PTH was combined with the anabolic intervention of anti-sclerostin antibody, both ultimate load and energy to failure exceeded values from normal animals (Figure 3). Enhanced vertebral mechanical properties have similarly been observed in ovariectomized animals treated with anti-sclerostin antibody [35]. Yet the response in these CKD animals differs from the long bone, where calcium plus anti-sclerostin antibody normalized, but did not significantly increase, ultimate load relative to NL animals [20] (Figure 5). These data illustrate the importance of normalizing PTH in treating the vertebral mechanical phenotype of CKD and highlight that anabolic therapy can be more effective at sites that have large proportions of both cortical and trabecular bone (such as the spine and femoral neck).

Structural mechanical properties are determined by bone mass, geometry/architecture, and the mechanical properties of the material (sometimes referred to as bone quality) [36]. Compromised bone mass and architecture are widely appreciated in CKD while the changes in bone quality have only recently begun to be explored [37,38]. We have shown, using both calculations of material properties from whole bone tests $[11,13,20]$ as well as by more direct measures [9] that long bones of CKD animals have reduced properties at the material level. This is associated with changes to both collagen and matrix hydration [39] although a cause-effect relationship has not been elucidated. The current work shows that high turnover, high PTH CKD disease compromises the mechanical properties of the material as both ultimate stress and toughness, are significantly lower than NL. This means that not only does the vertebra have lower bone mass but the bone mass that exists has compromised material properties relative to normal. Controlling PTH levels normalizes the material properties - contributing to the normalization of whole bone mechanical properties while treatments that did not affect PTH (zoledronic acid and anti-sclerostin antibody alone) had mixed effects on material properties.

The current experiment utilized zoledronate as a representative bisphosphonate to study in the setting of CKD. It is well accepted that each bisphosphonate has different biophysical properties of mineral binding strength and affinity to hydroxyapatite [40]. These properties 
affect many of the physical and physiological actions of the drugs including drug localization, speed of onset, degree of remodeling suppression, and speed of withdrawal [41]. It is unclear whether a different bisphosphonate would be more beneficial in the setting of CKD. Zoledronate has long lasting effects on remodeling suppression, more than 5 years following a single dose $[42,43]$ meaning that it would be difficult to washout the effect if needed. Lower affinity bisphosphonates, such as risedronate, have been shown to washout more rapidly than alendronate (a high affinity agent) [44] suggesting it may be more appropriate in the setting of CKD if concerns exist about treatment washout duration. More work with bisphosphonates are necessary to understand the details of their effects on bone, as well as the safety on the kidney, in the setting of CKD. It is possible that the there is diversity in the renal safety among the bisphosphonates but additional work is needed in this area [45]. In addition, despite efficacy of calcium in lowering PTH and improving serum calcium, in our previous publications we have observed increased arterial calcification suggesting that both efficacy and safety should be considered with any interventions.

In conclusion, the present study provides a clear picture of how progressive CKD, with and without interventions, affects vertebral bone properties. Since CKD-associated bone disease and fracture risk is significant at this site, it is important to recognize that response to treatment differs from long bones. Despite these differences, what is clear from this study is that reducing PTH is fundamental in normalizing mechanical properties. As such, any antiresorptive or anabolic bone therapies utilized in CKD should be examined in combination with therapies that suppress PTH (such as calcitriol and 1,25 vitamin D analogs and calcimimetics).

\section{ACKNOWLEDGEMENTS}

This work was supported by NIH grant AR058005 and funding from Novartis to SMM, NIH grant DK10093 to CLN and IUSM-CTSI training grant TR001107 to CLN. The authors would like to thank Dr. Michaela Kneissel for providing the anti-sclerostin antibody and input on dosing.

\section{REFERENCES}

1. Jamal SA, West SL, Miller PD. Fracture risk assessment in patients with chronic kidney disease. Osteoporos Int. 2011; 23:1191-8. [PubMed: 21901475]

2. Nickolas TL, McMahon DJ, Shane E. Relationship between moderate to severe kidney disease and hip fracture in the United States. J Am Soc Nephrol. 2006; 17:3223-32. [PubMed: 17005938]

3. Ensrud KE, Lui L-Y, Taylor BC, Ishani A, Shlipak MG, Stone KL, et al. Renal function and risk of hip and vertebral fractures in older women. Arch Intern Med. 2007; 167:133-9. [PubMed: 17242313]

4. Atsumi K, Kushida K, Yamazaki K, Shimizu S. Risk factors for vertebral fractures in renal osteodystrophy. American Journal of Kidney Disease. 1999

5. Nickolas TL, Stein EM, Dworakowski E, Nishiyama KK, Komandah-Kosseh M, Zhang CA, et al. Rapid cortical bone loss in patients with chronic kidney disease. J Bone Miner Res. 2013; 28:181120. [PubMed: 23456850]

6. Parfitt AM. A structural approach to renal bone disease. J Bone Miner Res. 1998; 13:1213-20. [PubMed: 9718188]

7. Eswaran SK, Gupta A, Adams MF, Keaveny TM. Cortical and trabecular load sharing in the human vertebral body. J Bone Miner Res. 2006; 21:307-14. [PubMed: 16418787] 
8. Shipov A, Segev G, Meltzer H, Milrad M, Brenner O, Atkins A, et al. The Effect of Naturally Occurring Chronic Kidney Disease on the Micro-Structural and Mechanical Properties of Bone. PLoS ONE. 2014; 9:e110057. [PubMed: 25333360]

9. Newman CL, Moe SM, Chen NX, Hammond MA, Wallace JM, Nyman JS, et al. Cortical Bone Mechanical Properties Are Altered in an Animal Model of Progressive Chronic Kidney Disease. PLoS ONE. 2014; 9:e99262. [PubMed: 24911162]

10. Iwasaki Y, Kazama JJ, Yamato H, Fukagawa M. Changes in chemical composition of cortical bone associated with bone fragility in rat model with chronic kidney disease. Bone. 2011; 48:1260-7. [PubMed: 21397740]

11. Allen MR, Chen NX, Gattone VH, Chen X, Carr AJ, LeBlanc P, et al. Skeletal effects of zoledronic acid in an animal model of chronic kidney disease. Osteoporos Int. 2012; 24:1471-81. [PubMed: 22907737]

12. Aoki C, Uto K, Honda K, Kato Y, Oda H. Advanced glycation end products suppress lysyl oxidase and induce bone collagen degradation in a rat model of renal osteodystrophy. Laboratory Investigation. 2013; 93:1170-83. [PubMed: 23979426]

13. Moe SM, Chen NX, Newman CL, Gattone VH II, Organ JM, Chen X, et al. A Comparison of Calcium to Zoledronic Acid for Improvement of Cortical Bone in an Animal Model of CKD. J Bone Miner Res. 2014; 29:902-10. [PubMed: 24038306]

14. Cowley BD, Grantham JJ, Muessel MJ, Kraybill AL, Gattone VH. Modification of disease progression in rats with inherited polycystic kidney disease. Yajkd. 1996; 27:865-79.

15. Cowley BD, Gudapaty S, Kraybill AL, Barash BD, Harding MA, Calvet JP, et al. Autosomaldominant polycystic kidney disease in the rat. Kidney Int. 1993; 43:522-34. [PubMed: 8455352]

16. Moe SM, Chen NX, Seifert MF, Sinders RM, Duan D, Chen X, et al. A rat model of chronic kidney disease-mineral bone disorder. Kidney Int. 2009; 75:176-84. [PubMed: 18800026]

17. Hoff S, Halbritter J, Epting D, Frank V, Nguyen T-MT, van Reeuwijk J, et al. ANKS6 is a central component of a nephronophthisis module linking NEK8 to INVS and NPHP3. Nat Genet. 2013; 45:951-6. [PubMed: 23793029]

18. Taskiran EZ, Korkmaz E, Gucer S. Mutations in ANKS6 Cause a Nephronophthisis-Like Phenotype with ESRD. Jasn. 2014

19. Brown JH, Bihoreau M-T, Hoffmann S, Kränzlin B, Tychinskaya I, Obermüller N, et al. Missense mutation in sterile alpha motif of novel protein SamCystin is associated with polycystic kidney disease in (cy/+) rat. J Am Soc Nephrol. 2005; 16:3517-26. [PubMed: 16207829]

20. Moe SM, Chen NX, Newman CL, Organ JM, Kneissel M, Kramer I, et al. Anti-Sclerostin Antibody Treatment in a Rat Model of Progressive Renal Osteodystrophy. J Bone Miner Res. 2014 n/a-n/a.

21. Parfitt AM, Drezner MK, Glorieux FH, Kanis JA, Malluche H, Meunier PJ, et al. Bone histomorphometry: standardization of nomenclature, symbols, and units. Report of the ASBMR Histomorphometry Nomenclature Committee. J Bone Miner Res. 1987; 2:595-610. [PubMed: 3455637]

22. Bouxsein ML, Boyd SK, Christiansen BA, Guldberg RE, Jepsen KJ, Müller R. Guidelines for assessment of bone microstructure in rodents using micro-computed tomography. J Bone Miner Res. 2010; 25:1468-86. [PubMed: 20533309]

23. Turner CH, Burr DB. Basic biomechanical measurements of bone: a tutorial. Bone. 1993; 14:595608. [PubMed: 8274302]

24. Jamal SA, Bauer DC, Ensrud KE, Cauley JA, Hochberg M, Ishani A, et al. Alendronate treatment in women with normal to severely impaired renal function: an analysis of the fracture intervention trial. J Bone Miner Res. 2007; 22:503-8. [PubMed: 17243862]

25. Nickolas TL, Leonard MB, Shane E. Chronic kidney disease and bone fracture: a growing concern. Kidney Int. 2008; 74:721-31. [PubMed: 18563052]

26. Dukas L, Schacht E, Stähelin HB. In elderly men and women treated for osteoporosis a low creatinine clearance of $<65 \mathrm{ml} / \mathrm{min}$ is a risk factor for falls and fractures. Osteoporos Int. 2005; 16:1683-90. [PubMed: 15933802] 
27. Danese MD, Kim J, Doan QV, Dylan M, Griffiths R, Chertow GM. PTH and the Risks for Hip, Vertebral, and Pelvic Fractures Among Patients on Dialysis. American Journal of Kidney Diseases. 2006; 47:149-56. [PubMed: 16377396]

28. Christov M, Pereira R, Wesseling-Perry K. Bone biopsy in renal osteodystrophy. Current Opinion in Nephrology and Hypertension. 2013:1. [PubMed: 23132368]

29. Malluche HH, Monier-Faugere MC. The role of bone biopsy in the management of patients with renal osteodystrophy. J Am Soc Nephrol. 1994; 4:1631-42. [PubMed: 8011971]

30. Ott SM. Bone histomorphometry in renal osteodystrophy. Semin Nephrol. 2009; 29:122-32. [PubMed: 19371803]

31. Spasovski GB. Bone biopsy as a diagnostic tool in the assessment of renal osteodystrophy. Int $\mathrm{J}$ Artif Organs. 2004; 27:918-23. [PubMed: 15636048]

32. Barreto FC, Barreto DV, Moyses RMA, Neves CL, Jorgetti V, Draibe SA, et al. Osteoporosis in hemodialysis patients revisited by bone histomorphometry: A new insight into an old problem. Kidney Int. 2006; 69:1852-7. [PubMed: 16612334]

33. Julian BA, Laskow DA, Dubovsky J, Dubovsky EV, Curtis JJ, Quarles LD. Rapid loss of vertebral mineral density after renal transplantation. N Engl J Med. 1991; 325:544-50. [PubMed: 1857390]

34. Burr DB. Why bones bend but don't break. J Musculoskelet Neuronal Interact. 2011:11.

35. Li X, Ominsky MS, Warmington KS, Morony S, Gong J, Cao J, et al. Sclerostin Antibody Treatment Increases Bone Formation, Bone Mass, and Bone Strength in a Rat Model of Postmenopausal Osteoporosis. J Bone Miner Res. 2009; 24:578-88. [PubMed: 19049336]

36. Seeman E, Delmas PD. Bone quality--the material and structural basis of bone strength and fragility. New England Journal of Medicine. 2006; 354:2250-61. [PubMed: 16723616]

37. Malluche HH, Porter DS, Monier-Faugere M-C, Mawad H, Pienkowski D. Differences in bone quality in low- and high-turnover renal osteodystrophy. Journal of the American Society of Nephrology. 2012; 23:525-32. [PubMed: 22193385]

38. Malluche HH, Porter DS, Pienkowski D. Evaluating bone quality in patients with chronic kidney disease. Nature Publishing Group. 2013; 9:671-80.

39. Changes in skeletal collagen crosslinks and matrix hydration in high and low turnover chronic kidney disease. 2014:1-27.

40. Nancollas GH, Tang R, Phipps RJ, Henneman Z, Gulde S, Wu W, et al. Novel insights into actions of bisphosphonates on bone: Differences in interactions with hydroxyapatite. Bone. 2006; 38:61727. [PubMed: 16046206]

41. Allen MR, Burr DB. Bisphosphonate effects on bone turnover, microdamage, and mechanical properties: what we think we know and what we know that we don't know. Bone. 2011; 49:56-65. [PubMed: 20955825]

42. Grey A, Bolland MJ, Horne A, Wattie D, House M, Gamble G, et al. Five years of anti-resorptive activity after a single dose of zoledronate - Results from a randomized double-blind placebocontrolled trial. Bone. 2012:1-5.

43. Reid IR, Lyles K, Su G, Brown JP, Walsh JP, del Pino-Montes J, et al. A single infusion of zoledronic acid produces sustained remissions in paget disease: Data to 6.5 years. J Bone Miner Res. 2011; 26:2261-70. [PubMed: 21638319]

44. Fuchs R, Phipps R. Recovery of trabecular and cortical bone turnover after discontinuation of risedronate and alendronate therapy in ovariectomized rats. Journal of Bone and. 2008

45. Pfister T, Atzpodien E, Bauss F. The renal effects of minimally nephrotoxic doses of ibandronate and zoledronate following single and intermittent intravenous administration in rats. Toxicology. 2003; 191:159-67. [PubMed: 12965119] 


\section{HIGHLIGHTS}

- An animal model of progressive kidney disease was studied to understand the effects of disease/treatment on spine properties.

- CKD negatively affected cortical and trabecular properties leading to compromised mechanical properties.

- Pharmacological treatments were effective in normalizing vertebral structure and mechanical properties only if the treatment reduced serum PTH. 


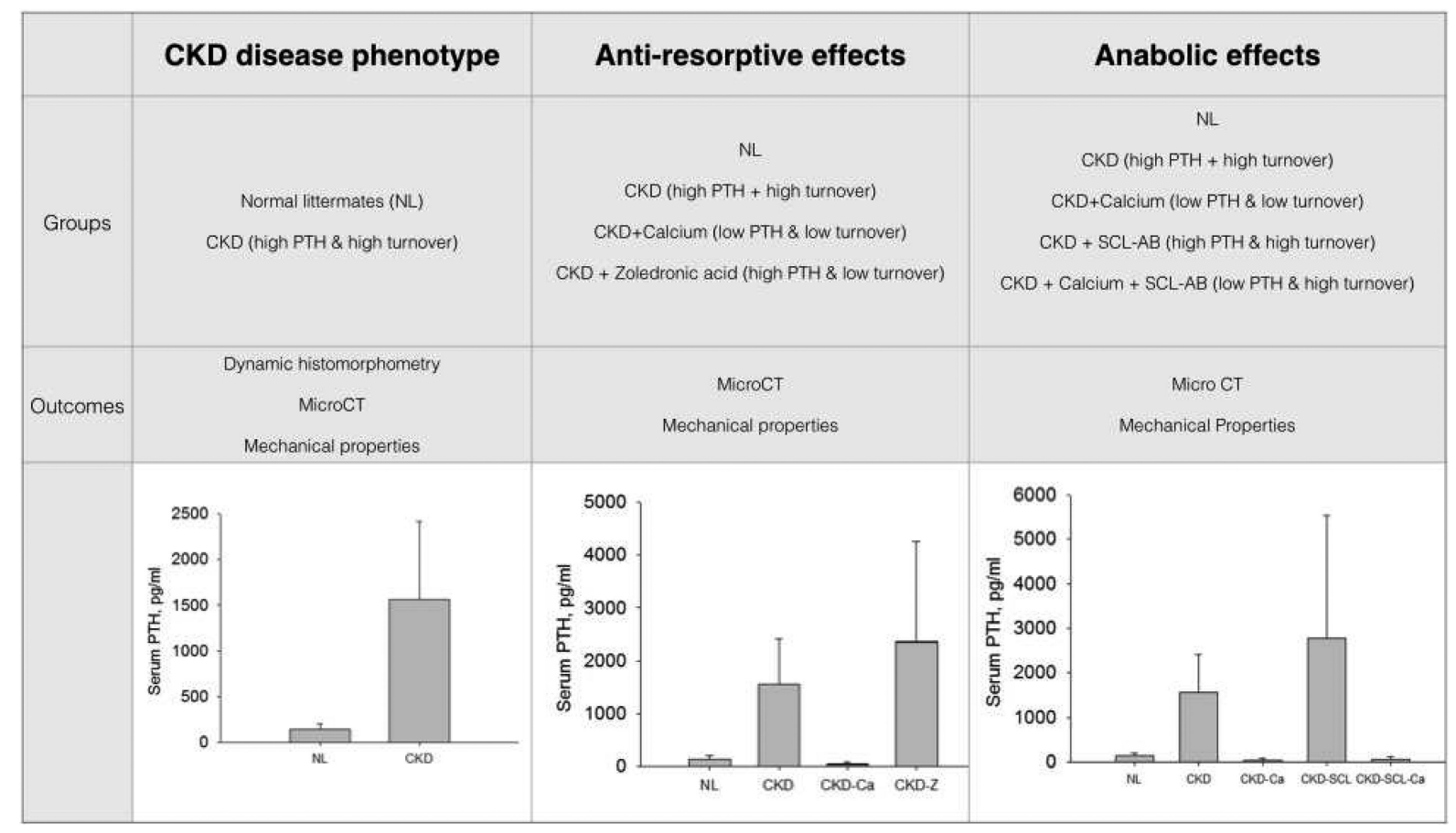

Figure 1.

Overview of experimental groups, outcome measures, and serum PTH levels for the planned comparisons. PTH values from previously published manuscript [20]. 

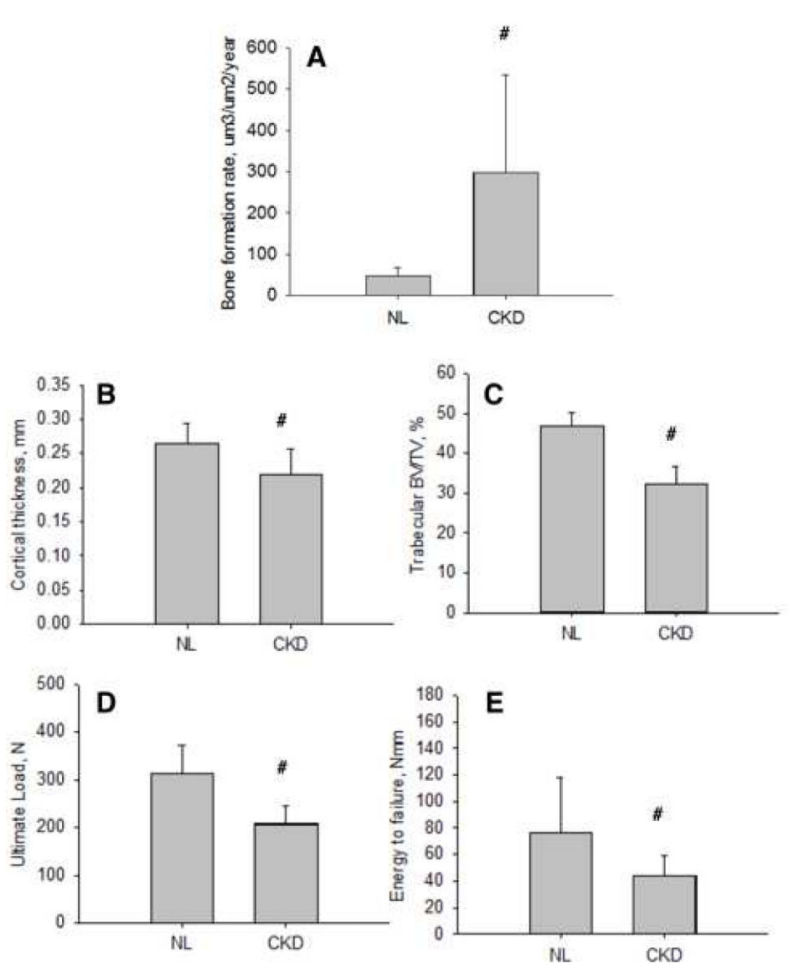
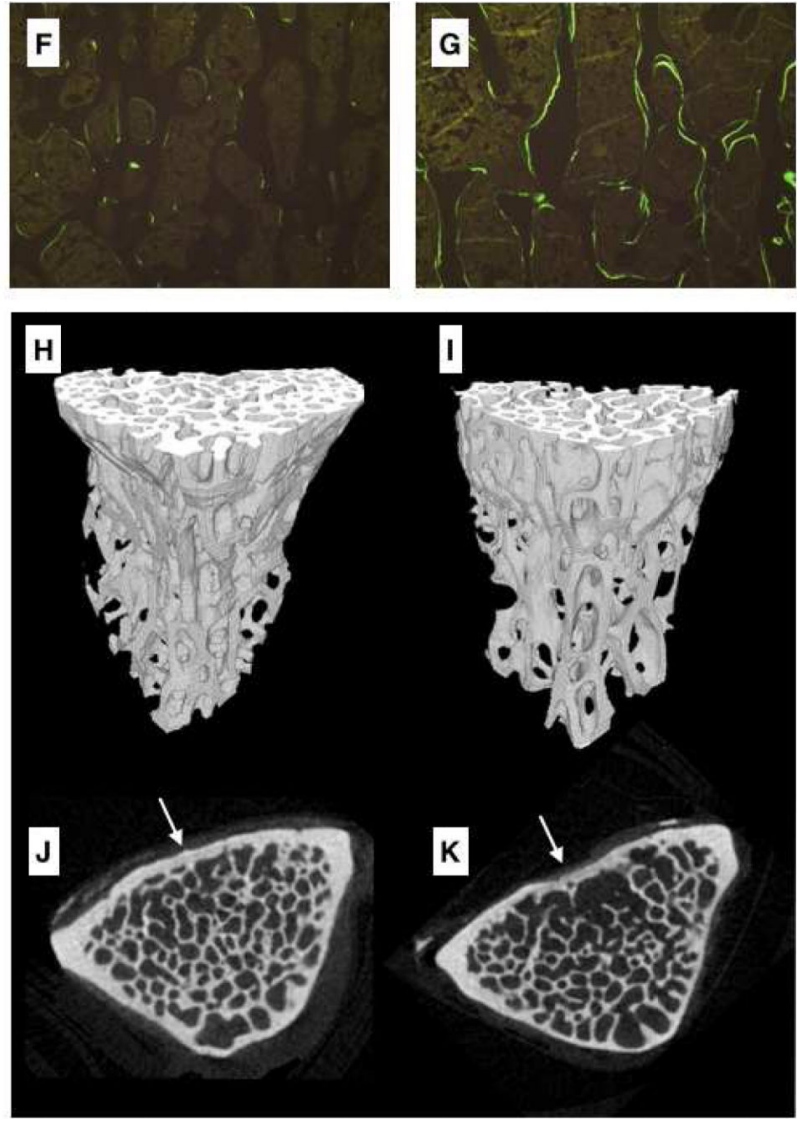

Figure 2.

Vertebral phenotype of animals with high-PTH CKD compared to normal littermates (NL). (A) Trabecular bone formation rate, (B) cortical thickness, (C) trabecular BV/TV, (D) ultimate load, and (E) energy to failure. Representative fluorochrome labeling images (taken from animal with remodeling values closest to group mean) from vertebral bone of NL (F) and CKD $(\mathrm{G})$ animals. Representative 3D reconstructions (taken from the animal with $\mathrm{BV} / \mathrm{TV}$ closest to the group mean) from trabecular bone in the vertebra of NL $(\mathrm{H})$ and CKD (I) animals. Representative cross-sections depicting region of cortical thickness assessment (in cortex noted by arrow) of NL (J) and CKD (K).\# $\mathrm{p}<0.05$ versus NL. 

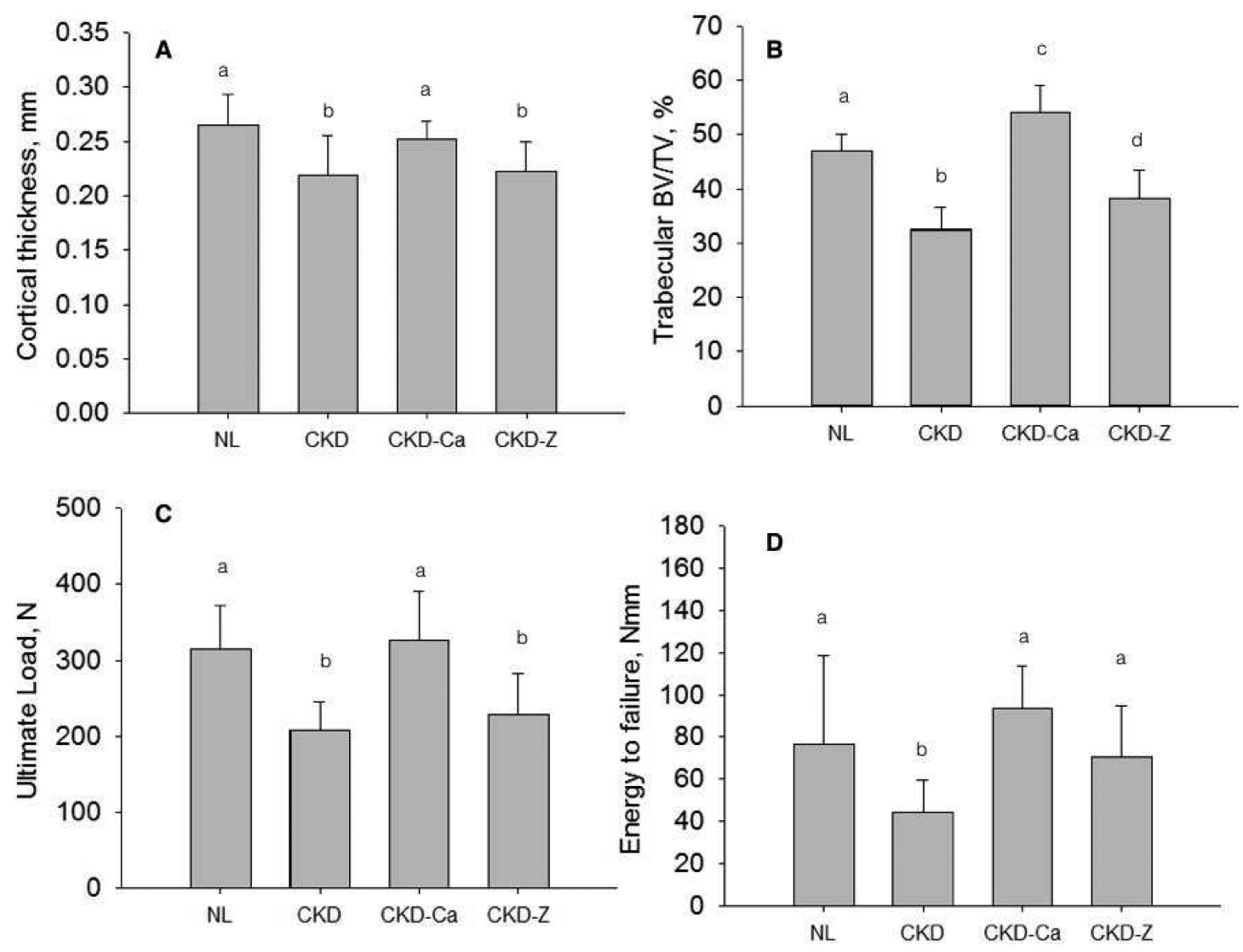

Figure 3.

Influence of reducing remodeling rate, with and without lowering $\mathrm{PTH}$, on the vertebral phenotype of animals with CKD. (A) cortical thickness, (B) trabecular BV/TV, (C) ultimate load, and (D) energy to failure. Groups with different letters are significantly different $(\mathrm{p}<$ 0.05 ). For all parameters, the overall ANOVA p value was $<0.05$. NL - normal littermates; CKD - animals with chronic kidney disease with high PTH levels; CKD-Ca - animals with chronic kidney disease treated with calcium to lower remodeling and PTH; CKD-Z animals with chronic kidney disease treated with zoledronic acid to lower remodeling. 

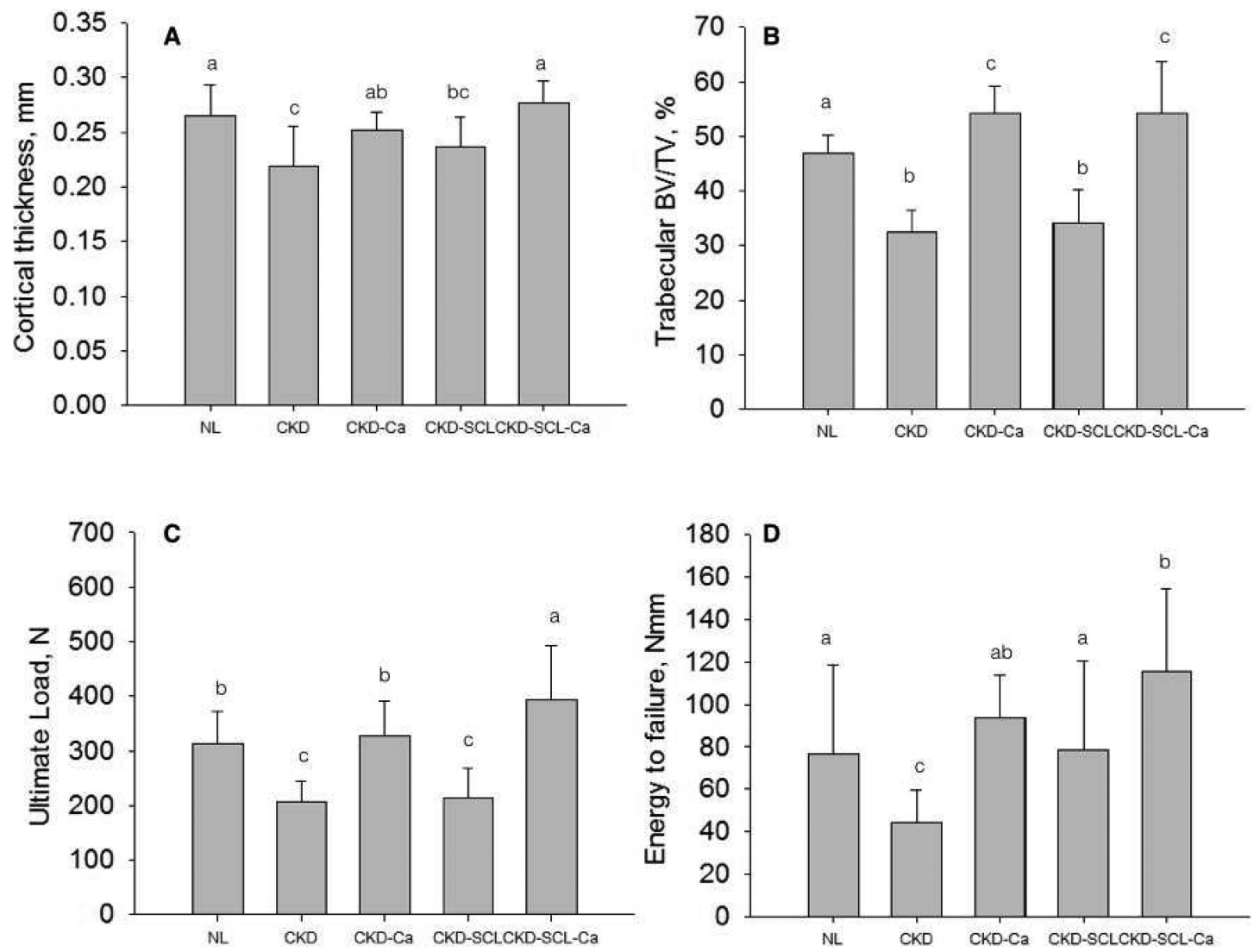

Figure 4.

Influence of anti-sclerostin antibody treatment, with and without lowering $\mathrm{PTH}$, on the vertebral phenotype of animals with CKD. (A) cortical thickness, (B) trabecular BV/TV, (C) ultimate load, and (D) energy to failure. Groups with different letters are significantly different $(\mathrm{p}<0.05)$. For all parameters, the overall ANOVA $\mathrm{p}$ value was $<0.05$. NL normal littermates; CKD - animals with chronic kidney disease with high PTH levels; CKD$\mathrm{Ca}$ - animals with chronic kidney disease treated with calcium to lower remodeling and PTH; CKD-SCL - animals with chronic kidney disease treated with anti-sclerostin antibody; CKD-SCL-Ca - animals with chronic kidney disease treated with anti-sclerostin antibody and calcium (to lower PTPH). 

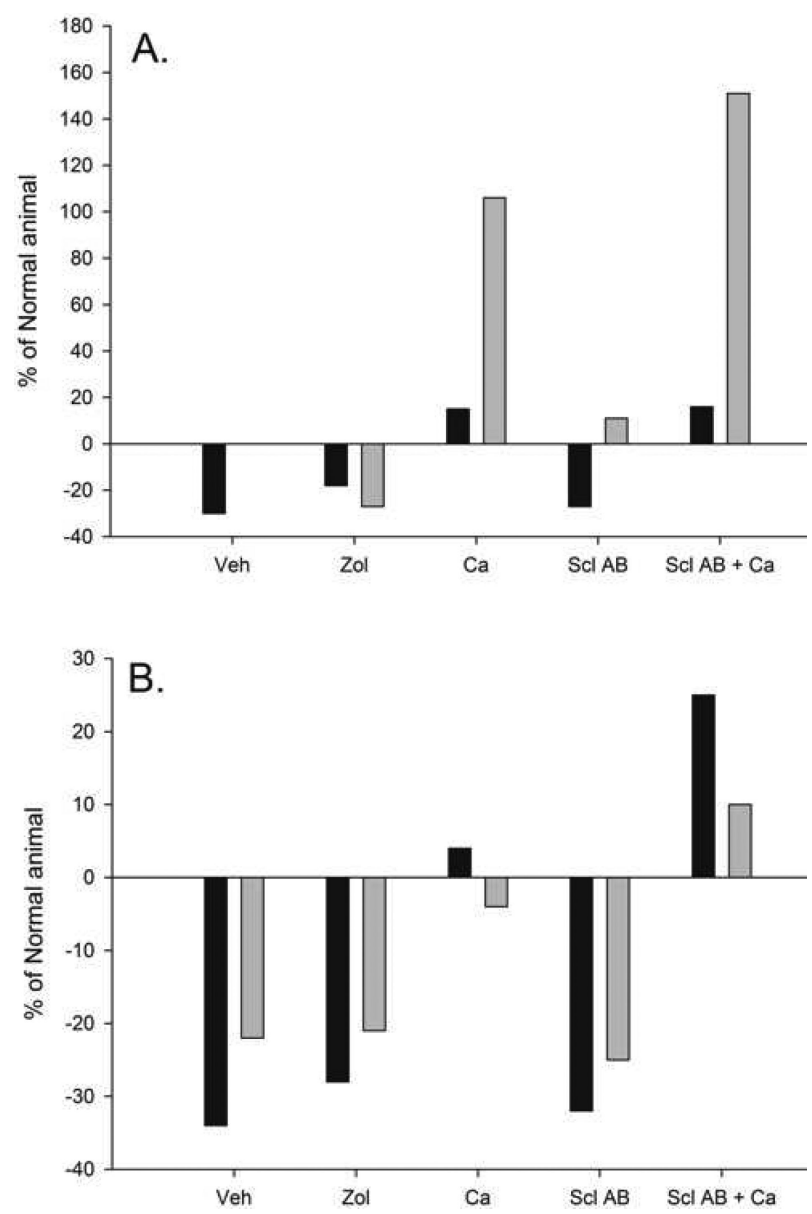

Figure 5.

Summary of vertebral and long bone response to disease and treatment response. (A) Trabecular bone volume of the vertebra (black bars) and proximal tibia (gray bars). (B) Ultimate load of the vertebra (trabecular and cortical bone together; black bars) and the femoral diaphysis (purely cortical bone; gray bars). Long bone data were pulled from previous publications in this same animal model. Data presented as percent of normal animals. 


\section{TABLE 1}

Mechanical phenotype of high PTH, high turnover CKD animals

\begin{tabular}{|l|c|c|c|c|}
\hline & Stiffness, N/mm & Ultimate stress, Pa & Modulus, Pa & Toughness, Pa \\
\hline $\mathrm{NL}$ & $1044 \pm 250$ & $30.8 \pm 3.9$ & $803 \pm 208$ & $0.95 \pm 0.41$ \\
\hline $\mathrm{CKD}$ & $829 \pm 332$ & $23.5 \pm 4.2$ & $728 \pm 290$ & $0.63 \pm 0.17$ \\
\hline $\boldsymbol{P}$ value & 0.035 & 0.002 & $\mathrm{NS}$ & 0.04 \\
\hline $\mathrm{CKD}-$ Calcium & $804 \pm 244$ & $37.8 \pm 9.9^{\mathrm{ab}}$ & $724 \pm 211^{\mathrm{b}}$ & $1.37 \pm 0.39^{\mathrm{ab}}$ \\
\hline $\mathrm{CKD}-$ Zoledronic acid & $646 \pm 345$ & $23.4 \pm 5.2^{\mathrm{a}}$ & $557 \pm 279^{\mathrm{a}}$ & $0.86 \pm 0.28$ \\
\hline ANOVA P value & 0.078 & 0.001 & 0.001 & 0.001 \\
\hline $\mathrm{CKD}-\mathrm{Scl} \mathrm{AB}$ & $477 \pm 237^{\mathrm{ab}}$ & $22.9 \pm 5.9^{\mathrm{ab}}$ & $426 \pm 200^{\mathrm{ab}}$ & $1.00 \pm 0.54^{\mathrm{b}}$ \\
\hline $\mathrm{CKD}-\mathrm{Scl} \mathrm{AB}+\mathrm{Calcium}$ & $1072 \pm 503$ & $34.1 \pm 8.5^{\mathrm{b}}$ & $709 \pm 282$ & $1.23 \pm 0.30^{\mathrm{b}}$ \\
\hline ANOVA P value & 0.002 & 0.001 & 0.016 & 0.006 \\
\hline
\end{tabular}

Data as mean \pm SD. Statistical comparisons were conducted as t-test (between NL and CKD), or ANOVA (between NL, CKD, CKD+Calcium, and $\mathrm{CKD}+$ Zoledronic Acid; and then between $\mathrm{NL}, \mathrm{CKD}, \mathrm{CKD}+\mathrm{Calcium}, \mathrm{CKD}+\mathrm{Scl} \mathrm{AB}$, and $\mathrm{CKD}+\mathrm{Scl} \mathrm{AB}+\mathrm{Calcium})$. Statistically different $(\mathrm{p}<0.05)$ values between $\mathrm{CKD}$ and $\mathrm{NL}$ are given in the t-test $\mathrm{p}$ values while differences in the ANOVA are noted relative to NL (a) and CKD (b). 


\section{Table 2}

Descriptive characteristics of animal groups.

\begin{tabular}{|l|c|c|c|c|}
\hline & Final body weight, $\mathbf{g}$ & Serum calcium, $\mathbf{~ g / d L}$ & Serum phosphorus, $\mathbf{~ g / d L}$ & BUN, $\mathbf{~ g / d L}$ \\
\hline $\mathrm{NL}$ & $554 \pm 41$ & $10.3 \pm 0.5$ & $4.9 \pm 0.9$ & $22 \pm 5$ \\
\hline $\mathrm{CKD}$ & $541 \pm 36$ & $9.0 \pm 2.0$ & $8.4 \pm 1.9$ & $49.2 \pm 8.2$ \\
\hline $\boldsymbol{P}$ value & NS & NS & $<0.001$ & $<0.001$ \\
\hline CKD - Calcium & $499 \pm 47^{\mathrm{ab}}$ & $11.9 \pm 1.5^{\mathrm{b}}$ & $7.0 \pm 1.2^{\mathrm{ab}}$ & $61.2 \pm 10.5^{\mathrm{ab}}$ \\
\hline CKD - Zoledronic acid & $531 \pm 41$ & $8.7 \pm 1.9$ & $7.6 \pm 1.9^{\mathrm{a}}$ & $56.2 \pm 16.5^{\mathrm{ab}}$ \\
\hline ANOVA P value & 0.04 & $<0.001$ & $<0.001$ & $<0.001$ \\
\hline CKD-Scl AB & $505 \pm 57$ & $9.9 \pm 2.0^{\mathrm{a}}$ & $9.4 \pm 3.9$ & $64.4^{\mathrm{a}} \pm 11.6^{\mathrm{ab}}$ \\
\hline CKD-Scl AB + Ca & $515 \pm 44$ & $11.1 \pm 2.7^{\mathrm{a}}$ & $7.9 \pm 2.1$ & $58.4 \pm 13.7^{\mathrm{ab}}$ \\
\hline ANOVA P value & NS & 0.018 & 0.002 & $<0.001$ \\
\hline
\end{tabular}

Data as mean \pm SD. Statistical comparisons were conducted as t-test (between NL and CKD), or ANOVA (between NL, CKD, CKD+Calcium, and $\mathrm{CKD}+$ Zoledronic Acid; and then between NL, CKD, CKD+Calcium, CKD+Scl AB, and CKD+Scl AB+Calcium). Statistically different ( $\mathrm{p}<0.05)$ values between CKD and NL are given in the t-test $\mathrm{p}$ values while differences in the ANOVA are noted relative to NL (a) and CKD (b). 